\title{
A Study on Structuring and Classification of Input Interaction
}

\author{
Younghwan Pan \\ Interaction Design, Graduate School of Techno Design, Kookmin University, Seoul, 136-702
}

\begin{abstract}
Objective: The purpose of this study is to suggest the hierarchical structure with three layers of input task, input interaction, and input device. Background: Understanding the input interaction is very helpful to design an interface design. Method: We made a model of three layered input structure based on empirical approach and applied to a gesture interaction in TV. Result: We categorized the input tasks into six elementary tasks which are select, position, orient, text, and quantify. The five interactions described in this paper could accomplish the full range of input interaction, although the criteria for classification were not consistent. We analyzed the Microsoft kinect with this structure. Conclusion: The input interactions of command, 4 way, cursor, touch, and intelligence are basic interaction structure to understanding input system. Application: It is expected the model can be used to design a new input interaction and user interface.
\end{abstract}

Keywords: Input interaction, Input task, Input device

\section{Introduction}

휴대폰은 화상통화, MP3 재생, 사진, 모바일 방송, 게임 등 다양한 기능을 수행할 뿐만 아니라, 와이파이, 와이브 로, 3G 망을 통해 인터넷에 연결을 할 수 있다. UI(User Interface, 사용자 인터페이스)의 표현 방법은 3D 모션 및 증강현실 및 가상현실을 표현할 수 있고, 입력의 경우에도 버튼과 음성인식, 터치 방식 이외에도 제스처 및 센서를 활 용한 다양한 입력 방식이 적용되고 있다. $\mathrm{PC}$ 의 경우는 네트 워크 및 브라우저 기술의 발전으로 SNS (Social Networking Service) 등 수많은 응용 프로그램 발전하고 있지만, 입력 기기는 키보드 및 마우스 이래로 큰 변화가 없다. 소수의 사용자를 위한 음성인식, 주시선을 활용한 입력기기는 있으 나, 대부분의 사용자는 키보드와 마우스를 사용하고 있다. TV는 디지털로 변화가 되면서 데이터 방송 및 다양한 콘텐 츠를 제공하고 있고, 입력은 리모콘이 주요 입력으로 활용되
면서, 제스처를 활용한 입력, 음성을 활용한 입력, 디바이스 제스처를 활용한 입력 등이 시도되고 있다. 리모콘도 IPTV (Internet Protocol Television)가 점차 확산되면서 이를 위한 리모콘은 기존의 리모콘과는 요구사항이 바뀌고 있다 (Kim, 2011). UI 표현 방식은 시스템의 발전으로 지속적으 로 다양해지고 발전하고 있지만 입력 부분은 제품별로 차이 가 있으나 표현 방식에 비해 발전의 속도가 더디다. 이 배경 에는 시스템은 지속적으로 발전을 하지만 신체는 발전하는 것이 아니어서, 입력의 시작인 신체의 변화는 적기 때문에 상대적으로 변화의 폭이 적다. 그렇다 하더라도 새로운 입력 방식은 UI에 많은 변화를 주기 때문에, 좀 더 혁신적이고 효 율적인 입력 방식에 대한 연구는 지속하고 있다. Goodwin (2009)은 다양한 입력 방식에 대해 조사 정리를 하였는데 터치 스크린, 소프트 버튼, 네 방향 커서 이동, 포인팅 디바 이스나 휠 등을 활용한 커서 이동, 센서를 활용한 방향 및 가속도의 입력, 노브와 다이얼, 스타일러스, 음성, 키보드, 발 페달과 같이 10 개의 입력 방식에 대한 장단점을 분석하였다.

Corresponding Author: Younghwan Pan. Interaction Design, Graduate School of Techno Design, Kookmin University, Seoul, 136-702.

Mobile: +82-10-3305-1011, E-mail: peterpan@kookmin.ac.kr

Copyright@2012 by Ergonomics Society of Korea(pISSN:1229-1684 eISSN:2093-8462). All right reserved.

(c) This is an open-access article distributed under the terms of the Creative Commons Attribution Non-Commercial License(http://creativecommons.org/licenses/by-nc/3.0/), which permits unrestricted non-commercial use, distribution, and reproduction in any medium, provided the original work is properly cited. http://www.esk.or.kr 
스마트폰을 기점으로 휴대폰에서의 입력 방식이 버튼에서 터치 스크린으로 바뀌고 있고, 터치 스크린 입력이 새로운 방식의 입력 방식은 아니지만, UI 표현 방식과 연계되어 좀 더 다양한 사용자 경험을 유도하고 있다. 또한 TV에서는 제 스처를 활용한 새로운 입력 방식의 시도가 이루어지고 있다. 제스처가 TV에서 적합한 방식인지 연구를 하기 위해서는 입력 방식을 구조적으로 이해하는 것이 필요하다.

Sharp 등 (2007)은 지시, 대화, 조작, 탐사 4가지 타입으 로 인터랙션을 개념화하였다. 이것은 단순히 디스플레이 상 에서의 인터랙션 뿐만 아니라 가상 공간과 실제 공간에서의 인터랙션 공간까지 고려를 하였다. 지시(instructing)는 사 용자가 명령어를 치거나, 메뉴에서 옵션을 선택하거나, 명령 어를 말하거나, 버튼을 누르는 등 시스템에게 지시를 하는 것이다. 대화(conversing)는 시스템이 대화 파트너처럼 반 응을 하는 것이다. 조작(manipulating)은 사물을 조작하는 것인데, 사용자의 물리적 환경에서의 조작 지식을 활용한다. 움직임, 선택, 열기, 닫기 등 뿐만 아니라 확대 축소 등도 가 능하다. 탐사(exploring)는 가상 공간이나 실제 공간에서 사 용자가 움직이는 것과 관련이 있다. Shneiderman(2000) 은 인터랙션 스타일을 5 가지 제안하였고, 각각의 장단점 에 대해 분석을 하였다. 메뉴 선택(menu selection), 양식 기입(form fill-in), 명령어(command language), 자연어 (natural language), 직접 조작(direct manipulation)의 5 가지 입력 스타일의 분류 체계를 제시하였다. 이와 유사 하게 Stone 등(2005)은 5개의 주요 인터랙션 스타일을 이야기했는데, 텍스트 기반의 명령어 입력인 명령어 라인 (command line), 사용자가 선택할 수 있는 옵션의 집합인 메뉴 선택(menu selection), 양식 형태로 정보를 기입하 는 양식 기입(form-fill), 사용자 인터페이스 대상에 직접 상호작용하는 직접 조작(direct manipulation), 사람들이 서로 상호작용 하는 것과 같은 방식으로 구현되는 의인화 (anthropomorphic)로 구조화 했고, 각각의 장단점에 대해 분석을 하였다. Ballard(2007)은 모바일 환경에서의 디바이 스를 대상으로 버튼, 음성, 음성과 버튼의 동시 사용, 시각 정보(바코드 인식, 사진 등) 와 버튼의 동시 사용으로 4 개의 입력 방식에 대해 연구하였다.

UI 디자인을 하는 경우에 출력 부분은 시스템의 목적과 정황에 따라 구성되기 때문에 일반적인 체계를 구축하는 것 이 어렵다. 즉 사용자 중심 디자인 관점에서 개념 디자인부 터 상세 디자인으로 반복 디자인 하는 것이 출력의 가장 대 표적인 화면에 표현되는 UI의 접근 방법이다. 또한 출력 부 문은 기술 발전에 의해 변화가 빠르게 나타난다. 이에 비해 입력 부분은 사용자에게서 출발하고, 특히 신체의 물리적 (음성 포함)인 움직임에 의해 시작되기 때문에 상대적으로 변화가 적다. 이는 입력의 체계를 구조화하는 것이 출력의
체계를 구조화 하는 것보다 상대적으로 단순하고 이해하기 쉽다. 최근에는 TV에서 음성, 제스처 등 다양한 형태의 입 력 방법을 재시도하고 있는데, 새로운 접근 방법으로 입력을 적용할 경우에 어떻게 인터페이스를 설계하는 것이 좋은지 를 이해할 때의 입력의 구조는 중요하다. 본 연구는 인터랙 션을 입력관점에서 분석하였고, 하나의 관점이 아니라 계층 적으로 구조화하였다.

\section{Structure of Input}

사람과 디지털 시스템의 인터랙션의 일반적인 절차는 Figure 1 과 같이 표명 단계, 변환 단계, 표현 단계, 평가 단 계의 4단계를 거친다(Kim, 2012). 이러한 구조는 사용자와 시스템의 관계를 유기적으로 표현해 주고 인터페이스의 기 본적인 구를 이해하는 데 도움을 준다. 하지만 이는 새로운 입력 방식이나 인터페이스를 설계할 때 구체적인 지침을 도 출하기에는 추상적인 구조이다.

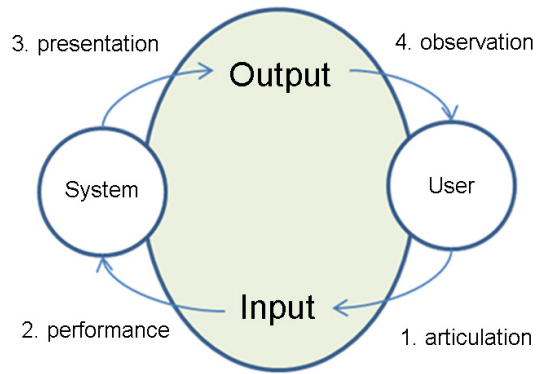

Figure 1. Structure of interaction

입력 체계를 입력의 목적, 방식, 도구라는 관점으로 계층 화하는 것이 인터페이스 설계할 때 구체적으로 도움을 줄 수 있다. 사용자는 입력 태스크라는 목적이 있고, 입력기기 라는 도구를 사용하고, 이를 사용하는 입력 인터랙션 방식이 있다(Figure 2).

입력 태스크는 실제로 출력에 영향을 미치는 부분이고, 시 스템에 성격에 따라 다양하기 때문에 기본적인 태스크를 이 해하는 것이 중요하다. Hinckley 등 (2009)은 복합 태스크 등을 언급했지만, 본 연구에서는 Foley 등(1984)이 제안한 태스크를 근거로 해서 기본적인 태스크 6 개를 분류하였다 (Table 1). 선택은 한 개를 선택할 수 있고, 여러 개를 선택 할 수 있다. 위치는 2 차원과 3 차원을 고려하였다. 방향은 3 차원에서의 회전을 고려하였고, 2 차원인 경우에는 2 개의 회전을 고려하면 된다. 경로는 위치의 변화 및 가속도로 구 


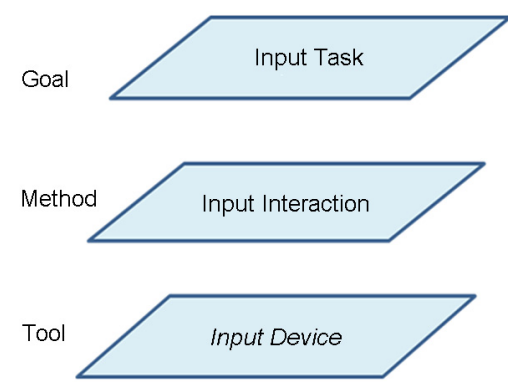

Figure 2. Hierachical structure of input

성하였고, 문자는 미리 정의된 문자를 대응시키는 것이고, 수치화는 숫자와 같이 절대적인 값을 가지는 경우와 상대적 인 값을 가지는 것으로 구성하였다. 이 외에도 더 많은 입력 태스크가 가능하나, 이 6가지를 가지고도 입력하는 목적을 기본적으로 구성할 수 있다.

입력 인터랙션은 입력기기와 완전히 분리할 수 없지만 분 리해서 이해하는 것은 UI 디자인에 도움이 된다. 새로운 입 력기기가 도출이 되는 경우라도 입력 인터랙션이 달라지지 않는 경우가 있다. 이는 UI 디자인을 다르게 할 필요가 없다 는 것이다. 같은 입력기기를 사용한다 하더라도 입력 인터랙 션을 변경하는 것이다. 이는 UI 디자인을 다시 해야 한다는 것이다. 입력기기는 눈에 보이고, 입력 인터랙션은 눈에 보 이지 않기 때문에, 입력 인터력션을 명확하게 정의 내리는 것은 $\mathrm{UI}$ 에 디자인에 도움이 된다.

입력기기는 리모콘, 마우스, 트랙볼, 마이크, 카메라, 음성 인식모듈 등 입력장치를 의미한다. 새로운 기술이 발달할수 록 점점 새로운 센서 및 기기가 발굴되고 있다. 이렇게 3 개 의 계층으로 입력을 이해하는 것은 UI에 디자인을 할 경우 에 구체적인 도움이 된다. 새로운 기술과 관련한 부분은 입

Table 1. Classification of input task

\begin{tabular}{c|l}
\hline Input task & Classification \\
\hline \multirow{2}{*}{ Select } & $\begin{array}{l}\text { - Single select } \\
\text { - Multi select }\end{array}$ \\
\hline \multirow{2}{*}{ Position } & $\begin{array}{l}-2 \text { dimensions } \\
\text { - } \text { dimensions }\end{array}$ \\
\hline \multirow{2}{*}{ Orient } & $\begin{array}{l}\text { - X rotation } \\
\text { - Y rotation } \\
\end{array}$ \\
\hline \multirow{2}{*}{ Path } & - rotation \\
\hline \multirow{2}{*}{ Text } & - Accelition variation \\
\hline \multirow{2}{*}{ Quantify } & $\begin{array}{l}\text { - Character } \\
\text { - Special character }\end{array}$ \\
\hline
\end{tabular}

력기기로 이해하고, 출력과 관련된 부분은 입력 태스크를 통 해 정의를 하고, UI 디자인 하는 경우는 입력 인터랙션을 잘 정의하면 된다. 새로운 입력기기가 개발이 되었을 경우에 이 세 가지를 잘 대응을 하면 입체적으로 입력기기를 이해할 수 있다.

\section{Classification of Input Interaction}

입력 인터랙션은 입력기기와 구분이 되는 입력하는 방식 이다. 본 연구에서는 명령어, 네 방향, 커서, 터치, 지능 인터 랙션 5 가지로 입력 인터랙션을 구분하였다. 이 구분은 인터 랙션간 완전히 배타적으로 분류되는 것은 아니지만, 휴대폰, $\mathrm{PC}, \mathrm{TV}$ 등 실제 제품들에 구성된 입력기기와 인터랙션을 분석하여 경험적으로 구성된 체계이다. 명령어 인터랙션은 시스템이 인지하는 명령어에 대응을 시키는 방식이다. 버튼 을 누를 수도 있고, 음성인식 기술을 통해 명령어를 전달할 수 있다. 네 방향 인터랙션은 네 방향 또는 상하좌우 버튼을 활용하여 화면장치의 포커스된 영역을 이동시켜 네비게이션 하는 방식이다. 아날로그 방식의 TV에서는 채널, 볼륨 중심 의 버튼 구성이었으면, 디지털 TV로 가면서, 채널, 볼륨 뿐 만 아니라, 메뉴, 네 방향 버튼, $\mathrm{OK}$, 뒤로 버튼 등 네비게이 션 버튼도 주요 버튼의 위치를 차지하게 되었다. 휴대폰도 스마트폰 이전에는 다양한 형태의 네비게이션이 있었지만, 각 회사들의 네비게이션이 네 방향 버튼과 확인과 취소로 수렴하고 있었다. 네 방향 인터랙션은 버튼을 활용한 입력에 서 기능이 많아지면서 생긴 필수불가결한 입력 방식으로 활 용되었고, 활용되고 있다. 터치 인터랙션은 커서 인터랙션과 유사하나, 화면장치에 네비게이션을 위해 포커스된 영역이 별도로 존재하지 않는 것이 일반적이다. 터치 인터랙션은 직 관적이고, 입력장치와 출력장치의 영역이 동일하다. 화면의 크기를 키우는 데 한계가 있는 휴대폰 영역에서 터치 입력은 출력장치의 크기를 최대화 할 수 있는 입력 방식이다. 지능 인터랙션은 앞의 네 가지 인터랙션과는 다른 기준으로 도출 하였고, 배타적으로 구성되지 않지만 실제로 이를 활용한 인 터랙션이 있고, 앞의 네 가지만으로는 설명하기가 어려워 구 성하였다. 지능 인터랙션은 시스템의 수준이 높아지면서 시 스템의 사람의 의도를 파악하여 반응하는 입력이다. 입력 인 터랙션도 세부적으로 분류하는 것이 UI 디자인에 도움이 된 다(Table 2).

\subsection{Command interaction}

명령어 인터랙션은 시스템과 대응 방식으로 구분할 수 있 
Table 2. Classification of input interaction

\begin{tabular}{c|l}
\hline Input iteraction & Classification \\
\hline \multirow{2}{*}{ Command } & $\begin{array}{l}\text { - Single select } \\
\text { - Multi select }\end{array}$ \\
\hline \multirow{2}{*}{4 Way } & $\begin{array}{l}\text { - 2 dimensions } \\
\text { - } 3 \text { dimensions }\end{array}$ \\
\hline \multirow{2}{*}{ Cursor } & - X rotation \\
& - Y rotation \\
& - Z rotation \\
\hline \multirow{2}{*}{ Touch } & - Position variation \\
& - Acceleration \\
\hline \multirow{2}{*}{ Intelligence } & - Character \\
& - Special character \\
\hline
\end{tabular}

다. $1: 1$ 대응은 입력과 시스템이 $1: 1$ 로 대응을 한다. 즉 한 번의 행위가 한 번의 명령어를 수행한다. N:1 대응은 $\mathrm{n}$ 개의 입력이 시스템의 명령어 1 개와 반응을 한다. 〈Ctrl $+\mathrm{A}>$ 등 과 같이 2 개의 입력이 하나의 명령어에 대응이 된다. 코드 키보드는 여러 개의 입력이 하나의 문자와 대응이 된다. $1: \mathrm{N}$ 대응은 하나의 행위가 여러 개의 명령어를 수행하는 것이다. 매크로 기능은 1 개의 입력이 여러 개의 명령어를 순차적으 로 수행을 하고, 소프트 키는 1 개의 행위가 1 개의 명령어를 수행하지만, 명령어가 정황에 따라 바뀌게 된다. 즉 1 개의 행위가 여러 개의 명령어에 대응이 되는 구조이다. 소프트키 의 대표적인 경우는 휴대폰에서 쓰이는 2 소프트 키와 3 소 트키가 있는데 Figure 3(a)는 3 소프트키의 사례이다. LSK (Left Soft Key), OK, RSK (Right Soft Key)로 구성된 3 소프트키가 적용된 휴대폰이다. Figure 3(b)는 리모콘에 컬러키가 적용이 되었는데, 이것이 소프트키의 개념이 적용 된 경우이다. 아날로그 TV에서는 유럽에서만 적용이 되었는 데, 디지털TV로 가면서 모두 채용해가는 추세이다.

\subsection{4 way interaction}

제품의 기능이 많아지면서, 명령어 인터랙션으로는 해결되 지 않아 GUI(Graphical User Interface)를 활용하여 표시 장치에서 미리 정의된 영역을 포커스된 영역을 움직이면서 네비게이션을 한다. 상하좌우 4 방향으로 움직이는 경우가 많 고, 초기에는 $\mathrm{OK}$ 버튼이 없었는데, 점차 $\mathrm{OK}$ 버튼이 포함된 방향으로 바뀌고 있다. OK 버튼이 없는 경우에는 메뉴 전개 방식에서 오른쪽 방향키를 선택하게 되면 방향 이동이 되는 경우와 선택이 되는 경우를 구성해서 디자인을 해야 한다. $\mathrm{OK}$ 가 있는지의 여부는 UI의 구성에 많은 영향을 준다. 네 비게이션 구성상 이전으로 가는 버튼을 별도로 할애하는 경 우가 많다.

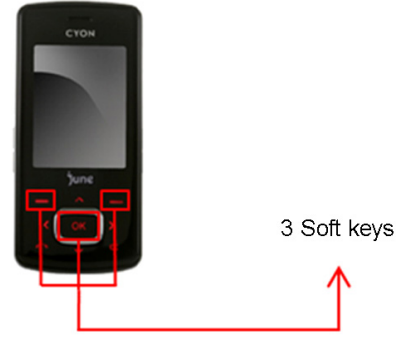

(a) 3 soft keys of a mobile phone

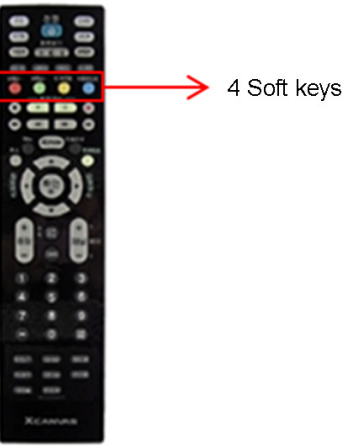

(b) 4 soft keys of a remote controller

Figure 3. Example of Soft keys(LG Products)

\subsection{Cursor interaction}

마우스가 대표적인 커서 인터랙션으로 커서의 움직임은 화면 상의 애플리케이션과 독립적으로 움직일 수 있다. 이는 멀티 태스킹에 아주 유리한 방식이고 $\mathrm{PC}$ 에 쓰이는 대표적 인터랙션 방식이다. 주시선을 이용하여 입력을 할 수 있는 경우도 있는데, 주시 선이 어느 특정 영역 이상에 머무르고 있으면 선택이 되기도 한다. 현재는 대부분 한 개의 커서로 만 구성되어 있지만, 화면이 커지고 여러 명이 동시에 사용 하는 경우에는 다중 커서로도 구성이 가능하다

\subsection{Touch interaction}

터치 인터랙션은 크게 한 손 터치와 멀티 터치로 구분할 수 있는데, 한 손 터치인 경우에는 탭, 롱탭, 더블탭, 드래그, 플릭 등이 있다. 상세한 터치 기술은 휴대폰에 적용이 되었 는데, 플랫폼(안드로이다, $\mathrm{iOS}$ ) 별로 약간의 터치의 적용 방 식은 다르지만, 유사하게 구현되어 있다. 멀티 터치인 경우 에는 다양한 형태의 인터랙션 태스크가 도출이 되는데, 한 손으로의 멀티 터치도 있지만 양 손으로 하는 멀티 터치도 가능하다. 


\subsection{Intelligence interaction}

앞의 네 가지 입력 인터랙션은 사용자가 하는 행위와 입력 기기의 동작 방식을 중심으로 분류를 한 것이면, 지능 인터 랙션은 시스템의 반응을 중심으로 한 입력 체계이다. 능동적 지능과 수동적 지능의 차이는 사용자가 입력의 의도가 있으 면 능동적 지능 인터랙션이고, 사용자의 의도가 없더라도 시 스템이 사용자를 의도를 파악하는 체계이면 수동적 지능 인 터랙션이다. 시스템의 판단 능력처리 능력이 필요한 인터랙 션이다.

\section{Case Study: Gesture Interaction in TV}

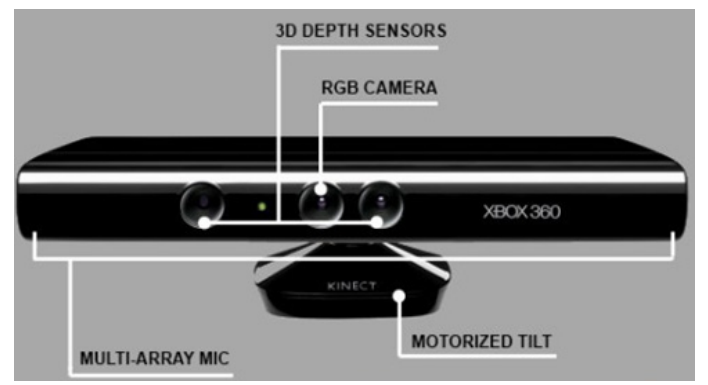

Figure 4. Microsoft kinect

$\mathrm{MS}$ 의 키넥트는 TV에 제스처를 활용하여, 적용시킨 경우 이다. 음성 부분도 있지만 GUI 메뉴 부분에서의 제스처를 중심으로 분석하면 Table 3 과 같다.

Table 3. Input Structure of Microsoft kinect

\begin{tabular}{c|c}
\hline Input structure & Microsoft kinect \\
\hline Input task & $\begin{array}{c}\text { Select(Single select) } \\
\text { Position(2 dimensions) } \\
\text { Orient } \\
\text { Path(Position variation) }\end{array}$ \\
\hline Input interaction & $\begin{array}{c}\text { Command interaction(1:1) } \\
\text { Cursor interaction }\end{array}$ \\
\hline Input device & $\begin{array}{c}\text { Kinect module } \\
\text { (3D depth sensors, RGB camera) }\end{array}$ \\
\hline
\end{tabular}

입력기기는 새로운 디바이스이지만 입력 인터랙션 측면에 서는 명령어를 전달하는 명령어 인터랙션과 마우스처럼 인 터랙션을 수행하는 것이다. 명령어는 사용자가 어떤 명령어
를 할 수 있는지를 알아야 하기 때문에, 실제로 명령어에 대 한 부분이 화면 상에 잘 표시가 되어야 한다. 이는 명령어의 종류를 많이 하기 쉽지 않다는 것을 의미한다. 제스처를 통 한 커서 인터랙션은 마우스에 비해 해상도와 반응속도가 좋 지 않기 때문에 수행도 측면에서의 장점은 없는 입력 인터랙 션이다. 결국 직관성과 재미에서 장점을 꼭 찾아야 하는 입 력 인터랙션 수행 방식이다. 입력 태스크는 선택되어야 하는 것이 있고, 입력이 3차원이 가능하더라도, GUI의 주요 네비 게이션 2 차원적으로 구성이 된다. 이처럼 입력 구조를 세 개 의 계층으로 분석을 하면, 입력 시스템을 활용한 UI에 도움 을 줄 수 있다.

\section{Conclusion and Discussion}

본 연구에서는 입력을 입력 태스크, 입력 인터랙션, 입력 기기 세 개의 계층으로 구분하여 각각을 분류하는 체계를 구축하였다. 입력 태스크는 6개의 단일 태스크로 구성하였고, 각각의 부 카테고리를 분류하였다. 마찬가지로, 입력 인터랙 션을 5 개의 인터랙션으로 구성하였고, 각각의 부 카테고리를 구성하였다. 입력 인터랙션은 경험적인 연구를 통해 구축한 분류 체계이고, 엄밀하게 배타적이고 독립적인 항목으로 구 성되지는 않았지만 실제 제품 연구에 도움이 될 수 있는 체 계로 구성을 하였다. 이것을 TV에서 키넥트 입력에 대해 분 석을 실시하였다.

추후 연구는 입력 태스크 부분을 출력 부분까지 연결하여 인터랙션의 확장 연구가 필요하고, 현재의 입력 인터랙션 체 계는 2 가지 기준이 섞여 있는 상황이어서, 좀 더 세밀한 분 류 체계에 대한 연구가 필요하다.

\section{Acknowledgements}

This work was supported by research program of Kookmin University in Korea.

\section{References}

Ballard, Barbara, Designing the Mobile User Experience, (pp. 56-58). Wiley, 2007.

Foley, J. D., Wallace, V. and Chan, P., "The Human Factors of computer 
graphics interaction techniques." IEEE Computer Graphics and Applications, 13-48, 1984.

Goodwin, Kim, Designing for the Digital Age, (pp. 441-443), Wiley, 2009. Hinckley, Ken, Human-Computer Interaction Fundamentals edited by Andrew Sears and Julie A. Jacko, (pp. 138-148), CRC Press, 2009.

Kim, J. H., Seok, J. H. and Han, J. W., "An Exploratory Study on the Problems of IPTV(Internet Protocol Television) Remote Controller Usability", Journal of Industrial Design, 5(3), 27-36, 2011.

Kim, J. W., Human Computer Interaction Introduction, (pp. 47-70), Ahngraphics, 2012.

Sharp, H, Rogers, Y and Preece, J., Interaction Design beyond HCI $2^{\text {nd }}$ edition, (pp. 64-83), Wiley, 2007.

Shneiderman, Ben, Human Computer Interaction: toward the year 2000 $2^{\text {nd }}$ edition written and edited by Ronal M. Baecker, Jonathan Grudin, Willian A. S. Buxton and Saul Greenberg, (pp. 401-410), Morgan Kaufmann Publishers, 1995.

\section{Author listings}

Younghwan Pan: peterpan@kookmin.ac.kr

Highest degree: $\mathrm{PhD}$, Department of Industrial Engineering, KAIST Position title: Assistant Professor, Department of Interaction Design, Graduate School of Techno Design, Kookmin University Areas of interest: User Experience Strategy, Service Design, Interaction Design

Date Received : 2012-07-17

Date Revised :2012-07-31

Date Accepted : 2012-08-03 